\title{
Correction to: Optical Metamaterials: Qualitative Models
}

\section{Correction to:}

\section{A. Chipouline and F. Küppers, Optical Metamaterials: Qualitative Models, Springer Series in Optical Sciences 211, https://doi.org/10.1007/978-3-319-77520-3}

In the original version of the book, department name of the volume editors was corrected from Technical University of Darmstadt to Darmstadt University of Technology, and the primary affiliation "Skolkovo Institute of Science and Technology, Moscow, Russia" was added to both the volume editors Prof. Arkadi Chipouline and Prof. Franko Küppers. The book has been updated with the changes. 